\title{
Erratum: Regulation of protein glycosylation and sorting by the Golgi matrix proteins GRASP55/65
}

\author{
Yi Xiang, Xiaoyan Zhang, David B. Nix, Toshihiko Katoh, Kazuhiro Aoki, Michael Tiemeyer \& Yanzhuang Wang
}

Nature Communications 4:1659 doi: 10.1038/ncomms2669 (2013); Published 3 Apr 2013; Updated 18 Sep 2013

This Article contains errors in Figs 6 and 8 that were introduced during the production process. In Fig. 6, images in panels a and b were inadvertently swapped with those in panels $\mathrm{c}$ and d, respectively. In Fig. 8d, double asterisks indicating statistical significance were omitted from the bar labelled ' $55+65$ '. Corrected versions of both figures appear below.
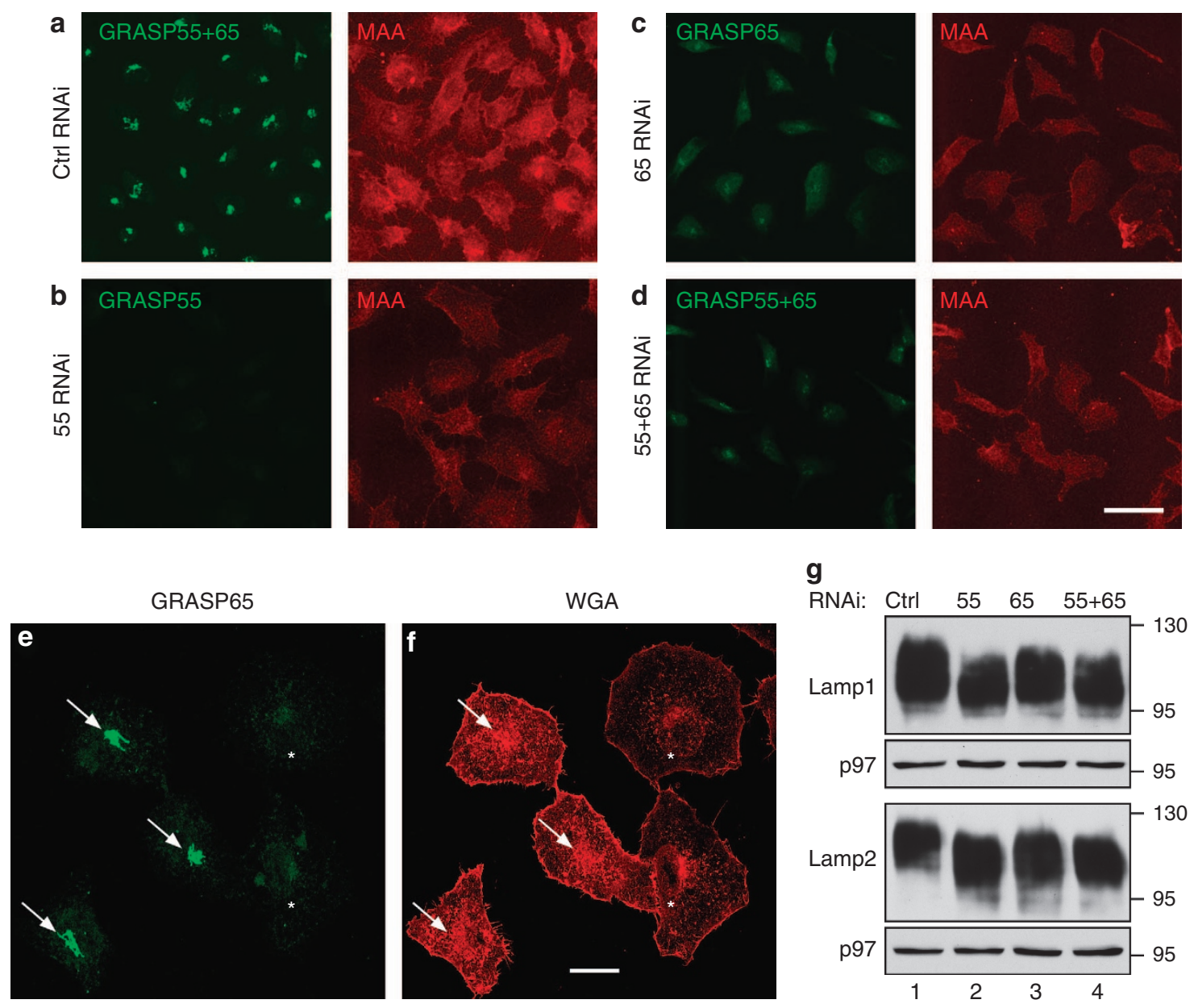

Figure 6 

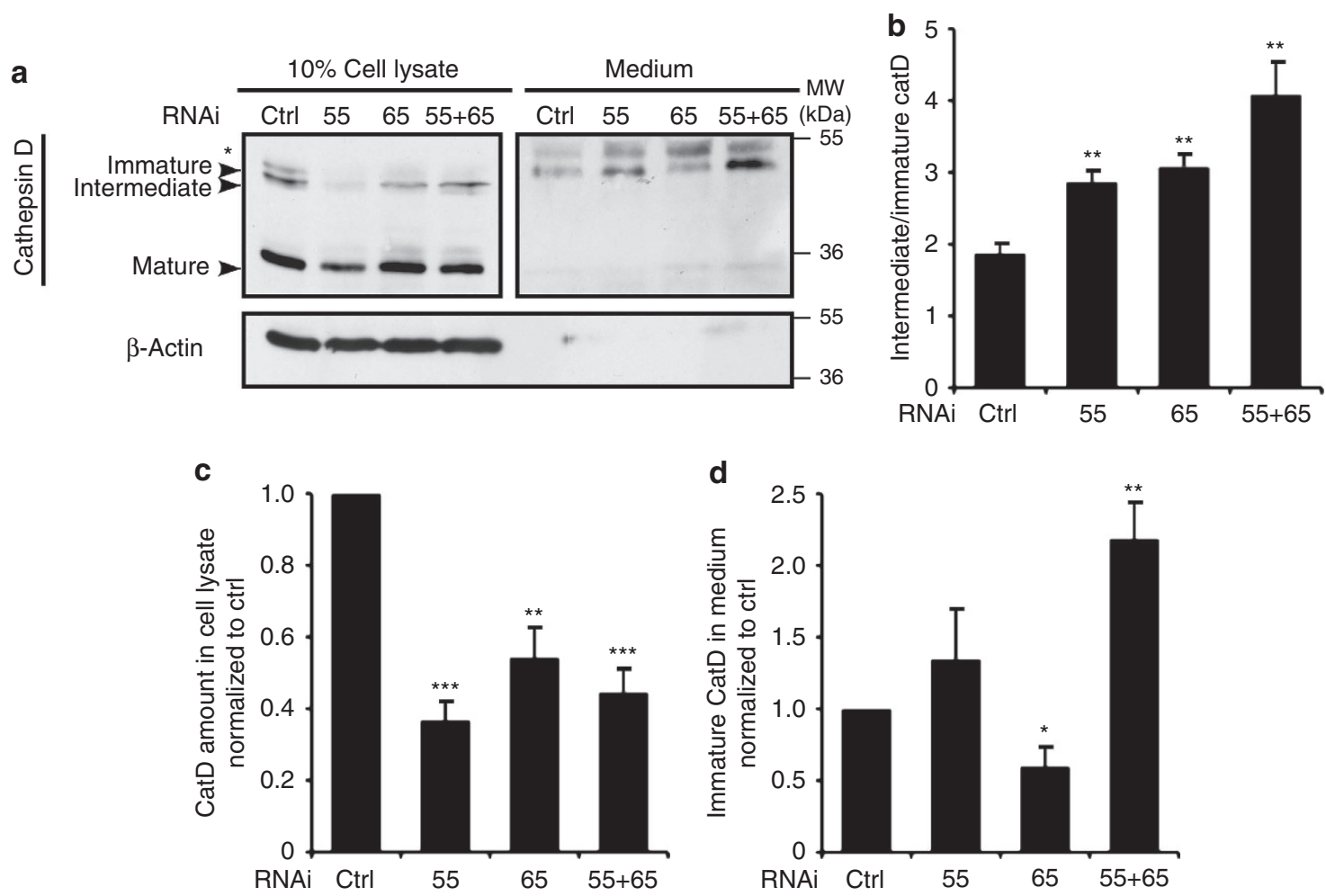

Figure 8 